\title{
Advances and Emerging Techniques in the Identification, Diagnosis and Treatment of Oral Candidiasis
}

\author{
Celeste M. Abraham* \\ Baylor College of Dentistry, The Texas A \& M University Health Science Center, 3302 Gaston Avenue, Dallas, TX \\ 75246, USA
}

\begin{abstract}
Oral candidiasis is a significant, frequent and familiar infection. Candida albicans is the most common fungal pathogen. It presents in both pathogenic and nonpathogenic states and has been able to develop a myriad of powerful mechanisms that allows for its thriving colonization and subsequent infection of the host under appropriate conditions. The objective of this paper is to evaluate advances in the cellular and molecular mechanisms involved in the diagnosis and identification of oral candidiasis and assess diverse novel treatment modalities for oral candidiasis in the clinical setting. Various cellular and molecular mechanisms have been employed to help elucidate the interactions between Candida organisms and their environment. Polymerase chain reaction analyses, Candida biofilm communication, and recombinant antigen techniques have been found to facilitate explanations of the endurance and continued existence of oral candidiasis, specifically C. albicans. Various modes of treatment, both traditional and innovative, have been utilized to manage oral candidiasis. These include, but are not limited, to the novel use of mucobuccal adhesives, denture liners, mouth rinse and toothpaste combinations as well as probiotic cheese.
\end{abstract}

Keywords: Oral candidiasis, emerging techniques, identification, treatment, diagnosis, cellular mechanism.

\section{INTRODUCTION}

Candida albicans is an important fungal pathogen that is not only capable of causing a localized intraoral Candida infection, but one that can permeate and initiate systemic effects as well. It is considered the most important of the Candida species and can be found in all areas of the mouth as well as on the surface of dentures [1-3]. The fungi that cause oral candidiasis are considered part of the normal flora of the mouth [4]. Intraoral candidiasis, particularly the prevalence of oropharyngeal candidiasis continues to escalate because of the increasing numbers of patients who are immunocompromised [5-7].

Oral candidiasis is caused by the fungal organism $C$. Albicans; it typically presents itself in a non-pathogenic state [8]. It is normally present in the mouth as the yeast form but under certain conditions can transform itself into the hyphal form which is pathogenic. There are certain environments which tend to set up this pathogenic state. These circumstances may include xerostomia, the use of antibiotics, systemic diseases, the presence of removable prostheses or the use of medications which suppress the immune system. Studies have suggested that in cases of denture stomatitis, Candidal proliferation occurs within denture plaque rather than within the mucosa; consequently, antifungal treatment should be targeted to both the denture in addition to the mucosa [8-10]. In a retrospective study of Candida colonization( count and species) in the presence and absence of removable dental prostheses, it was shown that a similar Candidal count and colonization pattern occurs on

*Address correspondence to this author at the Department of Periodontics, Baylor College of Dentistry, 3302 Gaston Avenue, Dallas, TX 75246, USA; Tel: 214-828-8467; E-mail: CAbraham@bcd.tamhsc.edu both oral tissues as well as on dental prostheses [11]. It was also noted that the treatment of oral candidiasis in denture wearers should also include the denture prostheses as well as the oral mucosa [11].

The diagnosis of oral candidiasis can be based on the clinical recognition of a particular form of Candida. Various clinical manifestations of oral candidiasis exist including pseudomembranous candidiasis, hyperplastic candidiasis (Candidal leukoplakia), atrophic candidiasis -acute and chronic (erythematous) and angular cheilitis [12, 13]. Pseudomembranous candidiasis may present as white plaques of hyphal aggregation. The underlying mucosa may be erythematous [12,13] (Fig. 4). Hyperplastic candidiasis has been also referred to as "Candidal leukoplakia". It clinically appears as white plaques that cannot be wiped away [12, 13] (Figs. 1, 2). Atrophic (Erythematous) candidiasis is a form where the lesions appear erythematous and often accompanied by dry mucosa. Chronic atrophic candidiasis is a form that is common to see in the hard palate and attached mucosa under a denture or removable partial denture [11-13]. This form is also known as denture stomatitis. Acute atrophic candidiasis commonly involves the dorsal tongue. It has been known by the term "median rhomboid glossitis" [12, 13]. Angular cheilitis is typically seen as red fissures at the corners of the mouth. It can be manifested as cracking and peeling and is often seen with other forms of candidiasis [12, 13] (Fig. 3). In addition to the clinical forms of Candida for diagnosis, there are also various species of oral Candida. Some of the species commonly seen intraorally are C. Albicans, C. glabrata, $C$. guillermondii, C. Krusei, C. parapsilosis, C. pseudotropicalis, C. stellatoidea, and C. tropicalis $[12,13]$.

The objective of this paper is to review advances in the cellular and molecular mechanisms in the diagnosis and 
identification of oral candidiasis and assess various novel ways of treating oral candidiasis in a clinical setting.

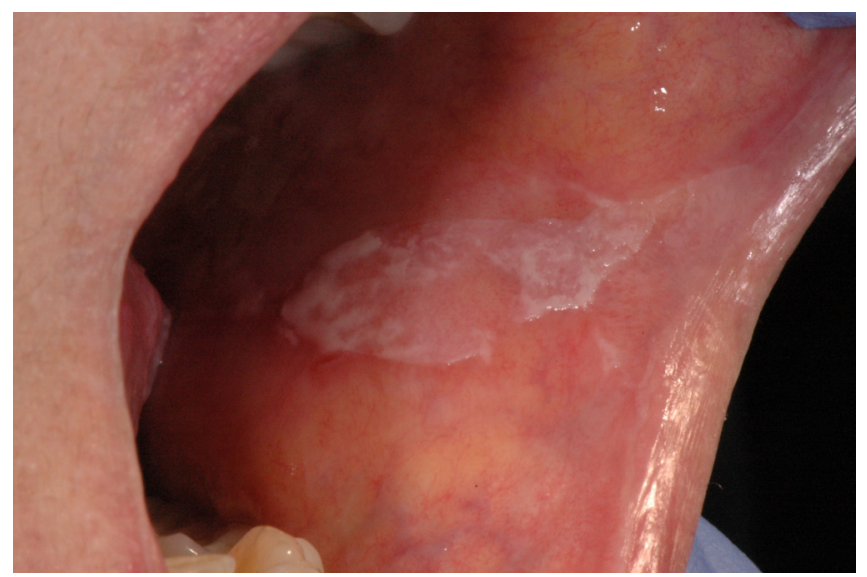

Fig. (1). Hyperplastic Oral candidiasis: Left buccal mucosa

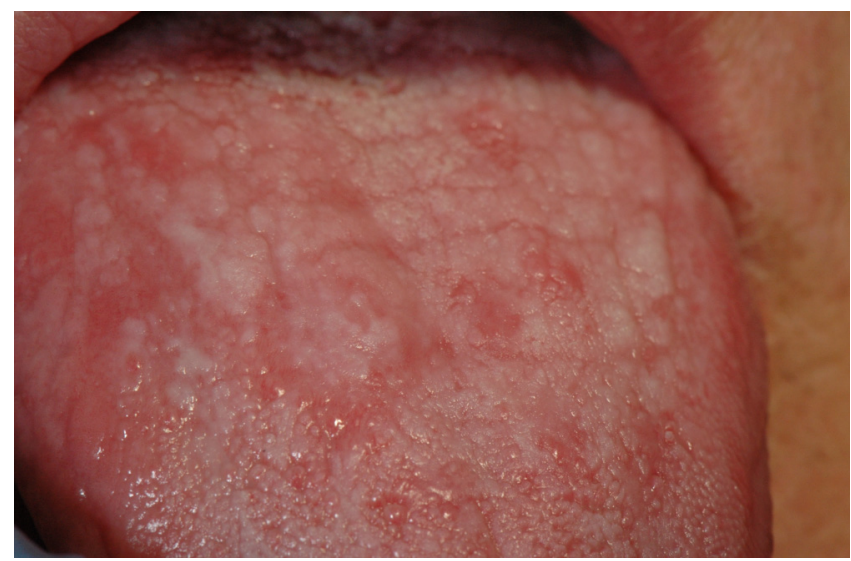

Fig. (2). Hyperplastic candidiasis: Dorsal tongue

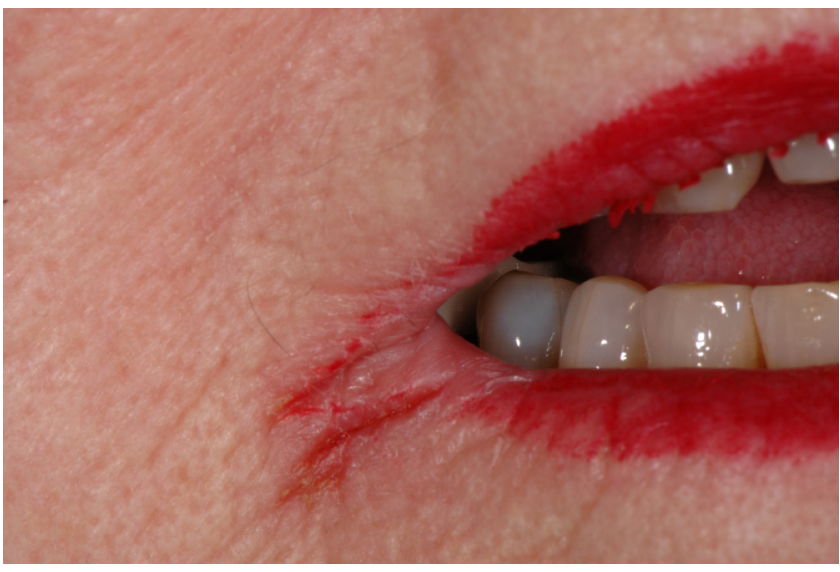

Fig. (3). Angular Cheilitis. Candidiasis at the right commissure.

CELLULAR AND MOLECULAR MECHANISMS UTILIZED IN ORAL CANDIDIASIS IN THE DIAGNOSIS AND IDENTIFICATION OF VARIOUS CANDIDA SPECIES

Oral Candida organisms have a manner in which they adhere to the oral mucosa as well as to non-oral surfaces (such as the denture) via the use of biofilms. Candida continues to exist intraorally because they are able to form biofilms that allow them to grow and flourish [14]. In the mouth, Candida exists in mixed biofilms, where they can live together with bacteria and successfully thrive in those environments [14]. It has been noted that it is the structure of the cell wall of Candida that displays properties that promote the formation of the biofilms, thereby allowing it to infiltrate the tissue and ultimately inhabit various organs in the body, including the oral mucosa $[14,15]$. Candida albicans is able to utilize special mechanisms that allow it to be able to switch back and forth from one form to another (from budding yeast form to pseudohyphal to hyphal forms) [14, 15]. For this reason, the formation of Candida biofilms creates serious clinical injurious effects because of their increased resistance to antifungal therapy; the cells within biofilms also carry the capability to resist immune defenses of the host [14]. This propensity to adhere to host cells of the epithelium is a virulence factor of species of oral Candida.

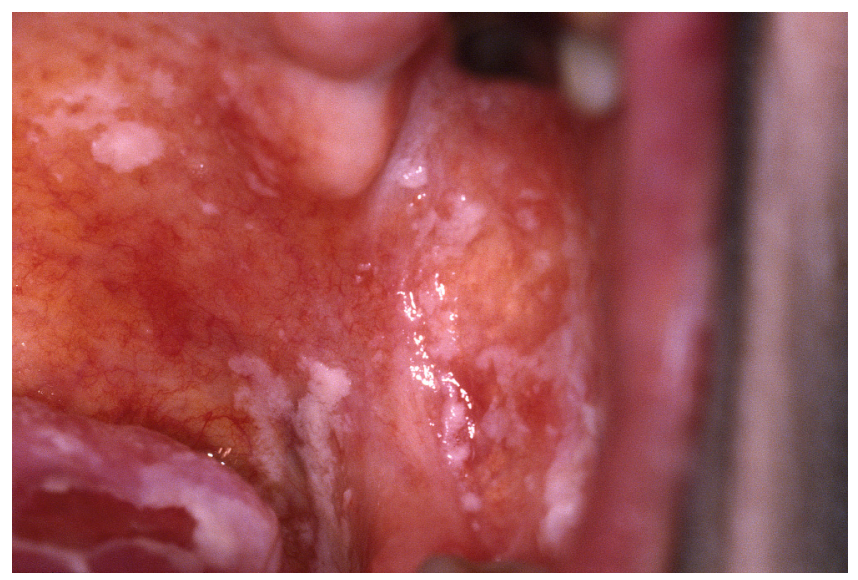

Fig. (4). Pseudomembranous candidiasis: Left side of the palate, left maxillary alveolar ridge and left buccal mucosa.

Additionally, it has been found, that Candida species and bacteria communicate with one another via a mechanism known as "quorum- sensing." [14, 16]. Candida and bacteria utilize signaling mechanisms where they have a direct effect on each other. C. albicans employs two major quorumsensing molecules known as tyrosol and farnesol. Tyrosol has the ability to stimulate the formation of hyphae in $C$. albicans while farnesol inhibits the shift from the yeast form to the hyphal form and regulates the morphology of the cell $[16,17]$. Quorum- sensing is a phenomenon where the accrual of signaling molecules enables a single cell to sense the number of bacteria/fungi that are present; this allows bacteria/fungi to manage and organize the way they behave so that they are able to respond quickly to their changing environments in order to sustain themselves.

\section{POLYMERASE CHAIN REACTION (PCR) FINGER- PRINTING}

Polymerase Chain Reaction (PCR) fingerprinting has been utilized to differentiate between various strains of $C$. Albicans and C. dubliniensis. This is a challenging problem because of the high degree of similarity in the phenotype between C. dubliniensis and C. Albicans [18]. Multiplex PCR has been used to identify various Candida species, particularly C. Albicans, C. glabrata, and C. tropicalis in a single sample [19]. Furthermore, PCR has been successfully used to identify C. dubliniensis in gingival fluid of healthy individuals with periodontal disease [20]. Tests were identified using conventional sampling methods as well as 
specific PCR assays. Results showed that $C$. dubliensis was isolated and identified in subgingival fluid of periodontal pockets of persons with healthy immune systems [20]. Realtime PCR and lucigenin-based chemiluminescence have demonstrated that Candida biofilms can exert resistance to many commonly employed antifungals in the clinical setting [21]. Real-time PCR chemistries allow for the detection of PCR amplification during the early phases of the reaction; measuring the kinetics during the early phases provides an advantage over the traditional PCR where agarose gels are used for the detection of PCR amplification at the final endpoint phase of the reaction [21]. C. Albicans genes have also been identified that regulate the communication between oral epithelial cells involved in oropharyngeal candidiasis and vascular endothelial cells [22]. These genes increase their interaction as they come into contact with epithelial or endothelial cells and as they endure the stresses that they likely encounter as they grow in the oropharynx and in the blood [22].

\section{RECOMBINANT DNA}

Use of recombinant antigens for the diagnosis of invasive candidiasis has been recently studied [23]. The detection of antibodies directed against antigens which are expressed on the $C$. albicans germ tube surface by indirect immunofluorescence has been shown to be valuable for diagnosis [23]. Among the Candida enzymes detected in sera of patients who have systemic candidiasis, enolase has been found to be highly diagnostic. Advances in molecular biological techniques have also allowed for the completion of the Candida albicans genome sequence. Overall, these molecular biology techniques have allowed the production of recombinant antigens which are useful for the discovery and identification of antibodies against them. It has been displayed that the detection of antibodies against purified and well-defined recombinant antigens allows for the diagnosis of Candidal organisms which are invasive in nature [23].

\section{RECONSTITUTED HUMAN ORAL EPITHELIUM (RHE)}

Host-fungus interactions were analyzed using a combination of microscopic, cellular and molecular techniques as well as an in vitro RHE (reconstituted human oral epithelium) model of oral candidiasis so at to scrutinize the Candida infection process [24]. It was found from this research analysis that morphology and adaptive response of Candida organisms play important roles in the way the host and the fungus respond to one another in the presence of an infection. Candida has also been recognized by the study of structural mucosal and innate immune cells [25]. The activation of mechanisms which are protective in nature in both immune and non-immune effector cells depends on appropriate recognition of pathogens. Pattern-recognition receptors expressed on host cells allow for the rapid recognition of pathogens [26]. C. Albicans has a an assortment of pathogen-associated molecular patterns; recognizing this array of patterns on the cell wall of $C$. Albicans is mediated through many pattern-recognition receptors such as the Toll-like receptors [27]. These Toll-like receptors are a separate family of pattern-recognition receptors that help to recognize microbes and eventually activate phagocytosis and inflammatory responses.
Various techniques have been used to identify Candida species. Multiplex PCR was used to quickly identify Candida species in samples of oral rinses without having to use an additional step of extraction in the process; ten species were identified in just a few hours [28]. Species that were identified included $C$. albicans, C. krusei, $C$. parapsilosis, C. famata, C. dubliniensis, C. kefyr, $C$. tropicalis, C. glabrata, C. guillermondii, and C. lusitaniae.

\section{IMMUNOLOGICAL METHODS}

Denture stomatitis is a very real and common problem encountered within the dental setting; a significant emphasis has been placed in this review in both the immunology as well as in the treatment because of the practical implications that this topic carries for the general as well as specialty dental practitioner.

In a denture-related stomatitis study, the main yeast strain isolated by far was C. Albicans. In the denture-related stomatitis group (versus the non-denture related stomatitis group), there were a greater variety of strains of Candida which were identified via microbiological sampling [29].

Candida mannan antigen detection was found to be a reliable as well as a sensitive method for the serologic diagnosis of systemic candidiasis [30]. The kit used in the study utilized an ELISA (Enzyme Linked Immunosorbent Assay) using polyclonal antibodies against Candida albicans mannan; the results revealed that the mannan assay had high positive results for Candida in comparison to the standard of the culture medium.

Denture stomatitis is a very real and common problem within the dental setting and a significant portion of this has been reviewed here with regards to immunologic aspects. It was noted in another investigation, that $C$. Albicans is the predominant fungal species isolated from those who wear dentures and that $C$. dubliniensis was isolated from the underlying mucosa but not from the prosthetic surface [31]. C. glabrata has been found to be an opportunistic pathogen in individuals who use long term immunosuppressants and broad-spectrum antibiotics-especially in the elderly [32]. This may be due to several factors, including decreased salivary gland hypofunction as well as $\mathrm{pH}$ changes noted in the elderly. The $\mathrm{pH}$ of the oral mucosa in the elderly appears to be more acidic than the oral $\mathrm{pH}$ of younger people [32].

\section{MICROBIAL CULTURE}

Identification of Candida species has been examined using CHROMagar [33]. CHROMagar ${ }^{\circledR}$ can differentiate $C$. albicans as green colonies, $C$. krusei as pink colonies and $C$. tropicalis as blue colonies. Candida species were isolated and identified using this agar from HIV patients with oropharyngeal candidiasis. C. albicans and C. krusei were identified with a few strains of $C$. tropicalis. A few strains of C. tropicalis generated colonies with a trace of a greenish color making it complicated to differentiate it from $C$. albicans.

In another identification and treatment analysis, fifty-five patients with complete upper dentures were examined before and after the use of a ten percent vinegar solution. Vinegar solution was used to disinfect their denture. Before the use of the solution, $C$. albicans, $C$. glabrata, C. krusei, $C$. 
lusitaniae, C. guillermondii, C. tropicalis, and $C$. parapsilosis were identified [34]. After the use of the vinegar solution, C. guillermondii, C. lusitaniae, and C. parapsilosis were not detected; all the other strains of Candida were still present. In a previous retrospective study of Candidal colonization and removable denture prostheses, forty-four patients exhibited various Candidal counts with respect to four groups of species: C. albicans, C. krusei, C. tropicalis, and unspecified Candidal species. The frequency of $C$. albicans was 87 percent with prostheses versus 90 percent without prosthesis; unspecified Candidal species was 58 percent versus 60 percent, $C$. krusei 14 percent with prosthesis versus 13 percent without prostheses, while the frequency of $C$. tropicalis was 6 percent for both [11].

\section{TREATMENT OF ORAL CANDIDIASIS}

Various traditional modes of treatment have been used to manage oral candidiasis. Treatment can be accomplished with the use of appropriate antifungal medications and routine disinfection of removable intraoral prostheses [35]. Both topical and systemic medications have been used. Topical agents include Nystatin oral suspension and nystatin oral powder as well as clotrimazole troches. Systemic antifungals can be given by prescription and include itraconazole, ketoconazole, and fluconazole. Mycostatin $\left({ }^{\circledR}\right.$ is a brand name for nystatin solution. Medications specifically for the treatment of angular cheilitis include nystatin ointment, miconazole cream $(2 \%)$, and clotrimazole cream (1\%). Disinfection of oral appliances can be accomplished via the use of Chlorhexidine and nystatin solution. (2\% chlorhexidine gluconate, Peridex) [36].

Newer antifungals in the prophylaxis and maintenance of oral candidiasis therapy for invasive fungal infections include Posaconazole, Ravucanazole, and Echinocandins such as Caspofungin, Micafungin, and Anidulafungin [37]. They all have been found to be less toxic antifungal alternatives to Amphotericin B. Posaconazole was approved in 2006 for the treatment of invasive Aspergillosis and Candida infections. Ravucanazole is highly effective against a variety of fungi, including Candida pathogens and has been used to treat esophageal candidiasis in HIV (human immunodeficiency virus) patients. Caspofungin has inhibitory effects against various Aspergillosis and Candida species. Micafungin has been shown to be highly effective against esophageal candidiasis and has fewer side effects than Caspofungin. Anidulafungin is the newest echinocandin and appears to be very effective against many Candida species in vitro. Anidulafungin was found also to be more efficacious in one study than the traditional form of systemic fluconazole for the treatment of invasive candidiasis [38].

Recent developments in the treatment of oral candidiasis include the use of probiotic bacteria which are known to reduce the growth of pathogenic microbes [39]. A recent study used cheese containing a mixture of probiotics such as Lactobacillus rhamnosus GG, Lactobacillus rhamnosus LC705, Propionibacterium freudenreichii and shermanii JS. The use of probiotic cheese revealed the reduction of the prevalence of oral Candida in the elderly and its use also allowed for the reduction of the prevalence of hyposalivation [39].
Another group of elderly individuals were evaluated for possible reduction of Candida overgrowth with the use of an amine fluoride-stannous fluoride mouth rinse and toothpaste combination [40]. The number of elderly with high Candida albicans counts decreased after use of the combination. Other Candida counts (non-albicans) were also low in the participants of the study.

In another novel application, fluconazole mucoadhesive buccal films were prepared and placed in the buccal mucosa where the drug was released over a five hour period and ultimately the disc eroded away. This topical method was used as another alternative to the traditional form of systemic fluconazole and seemed to reduce side effects of the medication that may have been encountered in the systemic form [41].

There has been current acceptance by the FDA for the miconazole Lauriad in a mucobuccal tablet form to treat oropharyngeal candidiasis in a once-a-day dosing format [42].

In a different treatment of denture stomatitis, dentureliner containing nystatin was placed for a period of one week. This was compared to a denture- liner filled with nystatin and left on the denture surface for four days. Dentures left with nystatin containing denture liner for four days had higher yeast counts than those that were left in place for one week [43]. Salivary yeast counts were much lower for individuals who had a denture liner that contained nystatin as the antifungal than individuals who did not use a denture liner containing an antifungal [43].

These and other recent advances have allowed the practitioner to treat various forms of Candida species in an effective manner in the clinical setting.

\section{REFERENCES}

[1] Lew MA. Diagnosis of Systemic Candida Infections. Ann Rev Med 1989; 40: 87-97.

[2] Coco BJ, Bagg J, Cross LJ, Jose A, Cross J, Ramage G. Mixed Candida albicans and Candida glabrata populations associated with the pathogenesis of denture stomatitis. Oral Microbiol Immunol 2008; 23(5): 377-83.

[3] Samaranayake YH, Cheung BP, Parahitiyawa N, et al. Synergistic activity of lysozyme and antifungal agents against Candida albicans biofilms on denture acrylic surfaces. Arch Oral Biol 2009; 54(2): 115-26.

[4] Lynch DP. Oral candidiasis. History, classification, and clinical presentation. Oral Surg Oral Med Oral Pathol 1994; 78(2): 189-93.

[5] Mean M, Marchetti O, Calandra T. Bench-to-bedside review: Candida infections in the intensive care unit. Critical Care 2008; 12: 204-13.

[6] Thaweboon S, Thaweboon B, Srithavaj T, Choonharuangdej S. Oral colonization of Candida species in patients receiving radiotherapy in the head and neck area. Quintessence Int 2008; 39(2): 52-7.

[7] Soysa NS, Samaranayake LP, Ellepola AN. Antimicrobials as a contributory factor in oral candidiasis- a brief overview. Oral Dis 2008; 14(2): 138-43.

[8] Davenport JC. The oral distribution of Candida in denture stomatitis. Brit Dent J 1970; 129: 151-6.

[9] Samaranayake LP, Keung LW, Jin L. Oral mucosal fungal infections. Perio 2000 2009; 49: 39-59.

[10] Dahlen G, Blomquist S, Carlen A. A retrospective study on the microbiology in patients with oral complaints and oral mucosal lesions. 2009; 15(4): 265-72.

[11] Abraham CM, Haghighat N, Beach MM, Al-Hashimi I. Candidal colonization in the presence and absence of removable dental prostheses. Tex Dent J 2007; 124(1): 94-101. 
[12] Zunt SL. Oral Candidiasis: Diagnosis and Treatment. The Journal of Practical Hygiene 2000; 9(5): 31-6.

[13] DeLong L and Burkhart NW. General and oral pathology for the dental hygienist. In Wolters Kluwer, Lippincott Williams \& Wilkins $1^{\text {st }}$ ed. 2008; pp: 349-51.

[14] ten Cate JM, Klis FM, Pereira-Cenci T, Crielaard W, de Groot PWJ. Molecular and cellular mechanisms that lead to Candida biofilm formation. J Dent Res 2009; 88: 105-15.

[15] Mukherjee PK, Zhou G, Munyon R, Ghannoum MA. Candida biofilm: a well-designed protected environment. Med Mycol 2005; 43: 191-208.

[16] Hornby JM, Jensen EC, Lisec AD, et al. Quorum sensing in the dimorphic fungus Candida albicans is mediated by farnesol. Appl Environ Microbiol 2001; 67(7): 2982-92.

[17] Chen H, Fujita M, Feng Q, Clardy J, Fink GR. Tyrosol is a quorum-sensing molecule in Candida albicans. Proc Natl Acad Sci USA 2004; 101(14): 5048-52.

[18] Neppelenbroek KH, Campanha NH, Spolidorio DM, Spolidorio LC, Seo RS, Pavarina AC. Molecular fingerprinting methods for the discrimination between Candida Albicans and Candida dubliensis. Oral Dis 2006; 12(3): 242-53.

[19] Liquori G, Di Onofrio V, Luvariello A, et al. Oral Candidiasis: a comparison between conventional methods and multiplex polymerase chain reaction for species identification. Oral Microbiol Immunol 2009; 24(1): 76-8.

[20] Jewtuchowicz VM, Mujica MT, Brusca MI, et al. Phenotypic and genotypic identification of Candida dubliensis from subgingival sites in immunocompetent subjects in Argentina. Oral Microbiol Immunol 2008; 23(6): 505-9.

[21] Seneviratne CJ, Wang Y, Jin L, Abiko Y, Samaranayake LP. Candida albicans biofilm formation is associated with increased antioxidative capacities. Proteomics 2008; 8(14): 2936-47.

[22] Park H, Liu Y, Solis N, et al. Transcriptional responses of Candida albicans to epithelial and endothelial cells. Eukaryot Cell. Aug 2009. Epub ahead of print. Published online ahead of print on 21 August 2009. Available from: http://ec.asm.org/cgi/content/abst ract/EC.00165-09v1: Accessed September 2, 2009

[23] Lain A, Eiguezabal N, Amutio E, Fernandez de Larrinoa I, Moragues MD, Ponton J. Use of recombinant antigens for the diagnosis of invasive candidiasis. Clin Dev Immunol 2008: 721950.

[24] Zakikhany K, Naglik JR, Schmidt-Westhausen A, Holland G, Schaller M, Hube B. In vivo transcript profiling of Candida albicans identifies a gene essential for interepithelial dissemination. Cell Microbiol 2007; 9(12): 2938-54.

[25] Villar CC, Dongari-Bagtzoglou A. Immune defence mechanisms and immunoenhancement strategies in oropharyngeal candidiasis. Expert Rev Mol Med 2008;10 Availablefrom http://www.pubmed central.nih.gov/articlerender.fcgi?artid $=2712880$. [cited: $2^{\text {nd }}$ Sept 2009].

[26] Mukhopadhyay S, Herre, J, Brown GD, Gordon S. The potential for toll-like receptors to collaborate with other innate immune receptors. Immunology 2004; 112: 521-30.

[27] Takeda K, Kaisho T, Akira S. Toll-like receptors. Ann Rev Immunol 2003; 21:335-76.
[28] Liguori G, Lucariello A, Colella G, De Luca A, Marinelli P. Rapid identification of Candida species in oral rinse solutions by PCR. J Clin Path 2007; 80: 1035-9.

[29] Figueiral MH, Azul A, Pinto E, Fonseca PA, Branco FM, Scully C. Denture-related stomatitis: identification of aetiological and predisposing factors - a large cohort. J Oral Rehab May 2007; 34(6): 448-55. Available from: http://www3.interscience.wiley. com/journal/118540440/abstract [cited: $2^{\text {nd }}$ Sept 2009]

[30] Kurita H, Kamata T, Zhao C, Narikawa J, Koike T, Kurashina K. Usefulness of a commercial enzyme-linked immunosorbent assay kit for Candida mannan antigen for detecting Candida in oral rinse solutions. Oral Surg Oral Med Oral Pathol Oral Radiol Endod 2009; 107(4): 531-4

[31] Marcos-Arias C, Vicente J, Sahand A, et al. Isolation of Candida dubliniensis in denture stomatitis. Arch Oral Biol 2009; 54(2): 12731 .

[32] Li L, Redding S, Dongari-Bagtzoglou A. Candida glabrata, an Emerging Oral Opportunistic Pathogen. J Dent Res 2007; 86; 20415 .

[33] Sivakumar VG, Shankar P, Nalina K, Menon T. Use of CHROMagar in the Differentiation of Common Species of Candida. Mycopathologia Epub Aug 2009; 167(1): 47-9. Available from: http://www.springerlink.com/content/4k040x4876 n63814/. [cited: $2^{\text {nd }}$ Sept 2009]

[34] Pinto TMS, Neves ACC, Leao MVP, Jorge AOC. Vinegar as an antimicrobial agent for control of Candida spp. in complete denture wearers. J Appl Oral Sci 2008; 16(6): 385-90.

[35] Arcangelo VP, Peterson AM. Pharmacotherapeutics for advanced practice: a practical approach. $2^{\text {nd }}$ ed. Wolters Kluwer, Lippincott Williams \& Wilkins 2005; pp: 756-59.

[36] Budtz-Jörgensen E, Löe H. Chlorhexidine as a denture disinfectant in the treatment of denture stomatitis. Europ J Oral Sci 2007; 80(6): 457-64.

[37] Petrikkos G, Skiada A. Recent Advances in antifungal chemotherapy. Int J Antimicrob Agents 2007; 30: 108-17.

[38] Kett DH, Cubillos GF. Anidulafungin in the treatment of patients with invasive candidiasis. Int J Antimicrob Agents 2008; 32 (Suppl 2): 99-102.

[39] Hatakka K, Ahola AJ, Yli-Knuuttila H, et al. Probiotics reduce the prevalence of oral Candida in the elderly--a randomized controlled trial. J Dent Res 2007; 86; 125-30.

[40] Meurman JH, Pärnänen P, Kari K, Samaranayake L. Effect of amine fluoride-stannous fluoride preparations on oral yeasts in the elderly: a randomised placebo-controlled trial. Gerodontol 2009; 26(3): 202-9

[41] Yehia SA, El-Gazayerly ON, Basalious EB. Fluconazole mucoadhesive buccal films: in vitro/in vivo performance. Curr Drug Deliv 2009; 6(1): 17-27.

[42] FDA Accepts Drug Application for Miconazole Lauriad to Treat Oropharyngeal Candidiasis. Today on pharmalive.com: 19Aug 2009: Available from: http://www.engelpub.com/News/Index.cfm? articleid $=646276$. [cited $2^{\text {nd }}$ Sept 2009]

[43] Geerts GA, Stuhlinger ME, Basson NJ. Effect of an antifungal denture liner on the saliva yeast count in patients with denture stomatitis: a pilot study. J Oral Rehabil 2008; 35(9): 664-9. 